\title{
Weyl points and exceptional rings with polaritons in bulk semiconductors
}

\author{
R. L. Mc Guinness (10) and P. R. Eastham (1) \\ School of Physics and CRANN, Trinity College Dublin, Dublin 2, Ireland
}

(Received 11 June 2020; revised 3 November 2020; accepted 4 November 2020; published 20 November 2020)

\begin{abstract}
Weyl points are the simplest topologically protected degeneracy in a three-dimensional dispersion relation. The realization of Weyl semimetals in photonic crystals has allowed these singularities and their consequences to be explored with electromagnetic waves. However, it is difficult to achieve nonlinearities in such systems. One promising approach is to use the strong coupling of photons and excitons, because the resulting polaritons interact through their exciton component. Yet topological polaritons have only been realized in two dimensions. Here, we predict that the dispersion relation for polaritons in three dimensions, in a bulk semiconductor with an applied magnetic field, contains Weyl points and Weyl line nodes. We show that absorption converts these Weyl points to Weyl exceptional rings. We conclude that bulk semiconductors are a promising system in which to investigate topological photonics in three dimensions, and the effects of dissipation, gain, and nonlinearity.
\end{abstract}

DOI: 10.1103/PhysRevResearch.2.043268

\section{INTRODUCTION}

Degeneracies in band structures are a key concept at the heart of recent developments in condensed-matter physics and optics [1]. Two-dimensional materials such as graphene possess Dirac points, where the dispersion is locally linear, which are responsible for many of their unique properties. In three-dimensional materials, Weyl points have been found in photonic [2] and electronic [3] band structures, providing lowenergy models of Weyl fermions. More generally, topological considerations mean that materials hosting degeneracies are the basis for realizing topological insulators and related effects such as robust edge modes. Such work is now also being extended to dissipative systems, such as photonic materials with gain and loss, described by non-Hermitian Hamiltonians [4]. In this case the singularities include exceptional points $[5,6]$ in parameter space, at which both the frequencies and lifetimes of the modes become degenerate. Rings of such exceptional points have been shown to emerge from Dirac points in photonic crystals [7]. In the three-dimensional case, Weyl points can become Weyl exceptional rings [8], which have a quantized Chern number and a quantized Berry phase. Like their counterparts in Hermitian systems, such non-Hermitian singularities give rise to interesting physical effects [9], including edge modes [10], unusual transmission properties, topological lasing, and Fermi arcs arising from half-integer topological charge [11].

Polaritons are exciton-photon superpositions that are formed by strong light-matter coupling in semiconductors [12,13]. Their half-matter half-light nature implies

\footnotetext{
Published by the American Physical Society under the terms of the Creative Commons Attribution 4.0 International license. Further distribution of this work must maintain attribution to the author(s) and the published article's title, journal citation, and DOI.
}

relatively strong nonlinearities, and this feature among others makes them an interesting system in which to study topological effects. Topological phases have been predicted [14-19] and observed [20] for polaritons formed from quantum-well excitons coupled to photons confined in microcavities. Topological lasing [21] and exceptional points [22] have also been studied. However, as microcavities and quantum wells are two-dimensional systems, phenomena such as Weyl points, Fermi arcs, and the three-dimensional topological phases [1] have not been considered.

In this paper, we report topologically nontrivial dispersion relations for polaritons propagating in three dimensions. We consider a bulk semiconductor in a magnetic field and show that the $p$-type structure of the valence band leads to intricate dispersion relations containing topologically protected degeneracies. In the absence of nonradiative losses there are eight sheets of the dispersion surface, which host Weyl points [2,3,23-25], for wave vectors along the field direction, and ring degeneracies, for wave vectors transverse to it. In the non-Hermitian case $[4,5,10]$, with absorption, we show that the Weyl points become Weyl exceptional rings, which can be reached by tuning the frequency and the angle between the propagation direction and the applied field. These results show that bulk semiconductors could be used to study topological effects in three spatial dimensions. Furthermore, bulk polariton lifetimes can be long since, unlike microcavity polaritons, they are not subject to radiative decay. They may therefore give access to the strongly interacting regime of topological photonics [1].

\section{METHOD}

\section{A. Exciton spectra}

We consider polaritons formed from $1 s$ excitons in direct band-gap zinc-blende semiconductors such as GaAs. These involve $p$-type valence-band states with $\Gamma_{8}$ symmetry and 


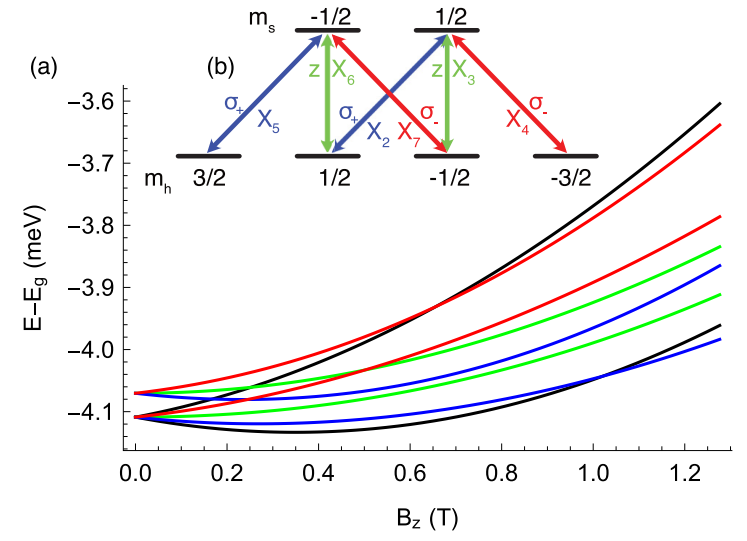

FIG. 1. (a) Calculated $1 s$ exciton energies relative to the band gap for GaAs with a magnetic field $B_{z} \in\left[0, B_{\max }\right]$ in the [001] direction. The line coloring indicates the polarization of each transition: right circular (red $/ \sigma_{-}$) and left circular (blue $/ \sigma_{+}$), with the field in the $x y$ plane, or linear in the $z$ direction (green $/ z$ ). The black curves are the spin-2 dark excitons. (b) Polarization and spin structure of the exciton transitions, in terms of the hole spin $m_{h}$ and electron spin $m_{s}$.

$s$-type conduction band states with $\Gamma_{6}$ symmetry. The combinations of the hole spin $m_{h}= \pm 3 / 2, \pm 1 / 2$ and the electron spin $m_{s}= \pm 1 / 2$ then give rise to eight exciton spin states, denoted $\left|X_{n}\right\rangle$ for $n=1, \ldots, 8$, with energies $E_{n}$.

To evaluate the polariton spectrum, we need the energies and polarizations of the exciton transitions. To obtain these, we diagonalize the effective Hamiltonian for the $1 s$ excitons given in Ref. [26]. The parameters in this effective Hamiltonian are related to the underlying electron-hole exchange parameters, Luttinger parameters, and $g$ factors. This approach treats the valence-band anisotropy, magnetic field, and electron-hole exchange as perturbations on a spherically symmetric electron-hole Hamiltonian [27]. The unperturbed wave function is of the usual hydrogenic form, with the binding energy $R_{0}=\mu e^{4} / 32 \pi^{2} \epsilon_{0}^{2} \epsilon^{2} \hbar^{2}$ and Bohr radius $a_{0}=$ $4 \pi \epsilon_{0} \epsilon \hbar^{2} / \mu e^{2}$, where $\mu^{-1}=m_{c}^{-1}+m_{v}^{-1} \cdot m_{v}=m_{0} / \gamma_{1}$ is the isotropic part of the effective mass for the valence band, related to the Luttinger parameter $\gamma_{1}$, and $m_{c}$ is the effective mass for the conduction band. For this perturbative approach to be valid the cyclotron energy must be small compared with the exciton binding energy $R_{0}$. We take the specific criterion given by Altarelli and Lipari [27],

$$
\gamma=\frac{\hbar \omega_{c}}{2 R_{0}}=\frac{e \hbar B}{2 \mu R_{0}} \leqslant 0.4
$$

to define the maximum field $B_{\max }$ of the perturbative regime. In the following we will consider the specific case of GaAs, with applied field $B_{\max }$ in the [001] direction, using the bandstructure parameters from Ref. [28]. For the electron-hole exchange parameters [26] we take $\Delta_{1}=-9.61 \mu \mathrm{eV}$ [29], and $\Delta_{0}=\Delta_{2}=0$. The exciton spectrum computed for these parameters is shown in Fig. 1(a). As expected, the magnetic field lifts the degeneracies of the eight electron-hole pair states. This splitting of the energies of the excitons will result in an anisotropic and multiply resonant optical susceptibility and hence a direction- and polarization-dependent polariton dispersion.

\section{B. Polariton Hamiltonian}

The topological singularities of the polariton dispersion arise from the polarization dependence of the exciton-photon coupling. In the Coulomb gauge the interaction between the vector potential and the electrons, from the Hamiltonian $\sum_{i}\left[\hat{\mathbf{p}}_{i}+e \hat{\mathbf{A}}\left(\hat{\mathbf{r}}_{i}\right)\right]^{2} /(2 m)$, is

$$
\hat{H}_{e p}=\frac{e}{m} \sum_{i} \sum_{\mathbf{k}, s} \sqrt{\frac{\hbar}{2 \epsilon_{0} \omega V}}\left[\hat{a}_{\mathbf{k}, s} \mathbf{e}_{\mathbf{k}, s} e^{i \mathbf{k} \cdot \hat{\mathbf{r}}_{i}}+\text { H.c. }\right] \cdot \hat{\mathbf{p}}_{i} .
$$

Here, the first sum is over the electrons, and the second is over the photon wave vectors, $\mathbf{k}$, and polarizations, $s$, with corresponding polarization vectors $\mathbf{e}_{\mathbf{k}, s} . \hat{a}_{\mathbf{k}, s}$ is the photon annihilation operator, $\omega=c|\mathbf{k}|$ is the photon frequency, and $V$ is a quantization volume. Thus we have the second-quantized Hamiltonian in the subspace of the eight $1 s$ exciton states, $\left|X_{\mathbf{k}, n}\right\rangle$,

$$
\hat{H}_{x p}=\frac{e}{m} \sum_{\mathbf{k}, s, n} \sqrt{\frac{\hbar}{2 \epsilon_{0} \omega V}}\left[\hat{X}_{\mathbf{k}, n}^{\dagger} \hat{a}_{\mathbf{k}, s} \mathbf{e}_{\mathbf{k}, s} \cdot\left\langle X_{\mathbf{k}, n}|\hat{\mathbf{p}}| 0\right\rangle+\text { H.c. }\right],
$$

where we have made the rotating-wave approximation. We have also neglected the diamagnetic term, proportional to $\hat{A}^{2}$, which is justified when treating the strong coupling of near-resonant modes [30]. Both the diamagnetic and counterrotating terms give nonresonant contributions which are perturbative in the ratio of the light-matter coupling strength to the exciton or photon energy, and hence small. There is, also, a resonant part of the diamagnetic term, which is accounted for in the observed value of the exciton energy. We note that the diamagnetic and counterrotating terms can be important away from resonance, being required, for example, to ensure the photon energy remains positive at long wavelengths. They are also important in the ultrastrong coupling regime, where the light-matter coupling becomes comparable to the transition energy.

In the envelope function approximation the matrix elements appearing in Eq. (3) are products of the matrix elements of the Bloch functions at $\mathbf{k}=0$ and the hydrogenic exciton wave functions $\chi_{m_{s}, m_{h}} F_{n}(\mathbf{r}=0)$. For the spatial part of the latter we take the unperturbed result $\left|F_{n}(0)\right|^{2} \approx 1 / \pi a_{0}^{3}$. For the spin part $\chi_{m_{s}, m_{h}}$ we note that at the field $B_{\max }$ we are considering, the Zeeman terms dominate over the electron-hole exchange. Thus the excitons are, to a good approximation, diagonal in the spin projections $m_{s}$ and $m_{h}$ [31]. Using the standard forms for the valence-band wave functions [32] and the Kane parameter $P$ [33-35], we then have $\left\langle X_{n}|\hat{\mathbf{p}}| 0\right\rangle=$ $\sqrt{V} m F_{n}^{*}(0)|P| \mathbf{v}_{n} / \hbar$, where $\mathbf{v}_{1}=\mathbf{v}_{8}=\mathbf{0}$ and

$$
\begin{array}{ll}
\mathbf{v}_{2}=\frac{1}{\sqrt{6}}\left(\begin{array}{l}
i \\
1 \\
0
\end{array}\right), & \mathbf{v}_{3}=\sqrt{\frac{2}{3}}\left(\begin{array}{l}
0 \\
0 \\
i
\end{array}\right), \quad \mathbf{v}_{4}=\frac{1}{\sqrt{2}}\left(\begin{array}{c}
-i \\
1 \\
0
\end{array}\right), \\
\mathbf{v}_{5}=\frac{1}{\sqrt{2}}\left(\begin{array}{l}
i \\
1 \\
0
\end{array}\right), & \mathbf{v}_{6}=\sqrt{\frac{2}{3}}\left(\begin{array}{l}
0 \\
0 \\
i
\end{array}\right), \quad \mathbf{v}_{7}=\frac{1}{\sqrt{6}}\left(\begin{array}{c}
-i \\
1 \\
0
\end{array}\right) .
\end{array}
$$

The states $\left|X_{1}\right\rangle \ldots\left|X_{4}\right\rangle$ correspond to excitons with electron spin $m_{s}=1 / 2$ and hole spin $m_{h}=3 / 2,1 / 2,-1 / 2,-3 / 2$, respectively, while $\left|X_{5}\right\rangle \ldots\left|X_{8}\right\rangle$ are the corresponding states with 
$m_{s}=-1 / 2$. Thus $\left|X_{1}\right\rangle$ and $\left|X_{8}\right\rangle$ are dark states, and the remaining transitions are either circularly polarized in the $x y$ plane or linearly polarized in the $z$ direction, as shown in Figs. 1(a) and $1(b)$.

Using these exciton matrix elements in Eq. (3), and approximating $\omega \approx E_{g} / \hbar$ in the prefactor of the coupling, we find for the exciton-photon Hamiltonian,

$$
\begin{aligned}
\hat{H}= & \sum_{\mathbf{k}}\left\{\sum_{s} \frac{\hbar c k}{\sqrt{\epsilon}} \hat{a}_{\mathbf{k}, s}^{\dagger} \hat{a}_{\mathbf{k}, s}+\sum_{n} E_{n} \hat{X}_{\mathbf{k}, n}^{\dagger} \hat{X}_{\mathbf{k}, n}\right. \\
& \left.+\frac{\hbar \Omega_{0}}{2} \sum_{s, n}\left(\mathbf{e}_{\mathbf{k}, s} \cdot \mathbf{v}_{n}\right) \hat{X}_{\mathbf{k}, n}^{\dagger} \hat{a}_{\mathbf{k}, s}+\text { H.c. }\right\},
\end{aligned}
$$

where $k=|\mathbf{k}|$ and we use a Rabi splitting

$$
\hbar \Omega_{0}=\sqrt{\frac{2 e^{2}|P|^{2}}{\epsilon_{0} \epsilon E_{g} \pi a_{0}^{3}}}
$$

to quantify the light-matter coupling in the material.

\section{Polariton spectra}

In the following we will consider the polariton spectrum, which we obtain from the Heisenberg equations of motion for the exciton and photon annihilation operators by looking for solutions with time dependence $e^{-i \omega t}$, i.e., setting $\hat{a}(t)=e^{-i \omega t} \hat{a}(0)$ and similarly for the exciton operators. We specify the wave-vector direction in terms of the polar coordinates $\theta, \phi$, with the field direction and the [001] crystal axis corresponding to $\theta=0$. For the photon polarization we use the circularly polarized states, $\mathbf{e}_{\mathbf{k}, \pm}=\left(\mathbf{e}_{\mathbf{k}, \theta} \pm i \mathbf{e}_{\mathbf{k}, \phi}\right) / \sqrt{2}$, constructed from the linearly polarized basis transverse to $\mathbf{k}$,

$$
\mathbf{e}_{\mathbf{k}, \theta}=\left(\begin{array}{c}
\cos \theta \cos \phi \\
\cos \theta \sin \phi \\
-\sin \theta
\end{array}\right), \quad \mathbf{e}_{\mathbf{k}, \phi} \quad=\left(\begin{array}{c}
-\sin \phi \\
\cos \phi \\
0
\end{array}\right)
$$

Since there are two photon polarizations and six excitons (discounting the irrelevant dark states), this procedure gives an $8 \times 8$ Hamiltonian matrix, $H_{8}$, with elements dependent on the wave-vector magnitude and direction. The form of this Hamiltonian is given explicitly in the Appendix. We determine the polariton dispersion $\omega(k, \theta, \phi)$ by finding the eigenvalues, $\omega$, of $H_{8}$ numerically.

While the dispersion $\omega(k, \theta, \phi)$ is given by the eigenvalues of $H_{8}$, there is another approach to analyzing the topological singularities of the polariton spectrum, in terms of the function $k(\omega, \theta, \phi)$. This latter function provides a natural description of optics at a fixed frequency and is related to constructs such as the refractive index surface of classical optics [36-38]. For example, a radial plot of $k$ at some fixed frequency over angles gives a contour (in $k$ space) of the dispersion relation $\omega(k)$. The normal to such an isofrequency surface is therefore the group velocity, $\nabla_{\mathbf{k}} \omega$, controlling the refraction direction at that frequency. While the two functions $\omega(k, \theta, \phi)$ and $k(\omega, \theta, \phi)$ are equivalent in the absence of dissipation, we shall see that they have some differences in its presence, and we therefore consider both representations in the following.

To obtain the dispersion in the form $k(\omega, \theta, \phi)$, we eliminate the exciton amplitudes from the Heisenberg equations of motion. This leads to a two-dimensional eigenproblem for the photon amplitudes,

$$
\left[(\omega-c k / \sqrt{\epsilon}) \delta_{s s^{\prime}}-\frac{\Omega_{0}^{2}}{4} \sum_{n} \frac{\left(\mathbf{e}_{\mathbf{k}, s}^{*} \cdot \mathbf{v}_{n}^{*}\right) \cdot\left(\mathbf{e}_{\mathbf{k}, s^{\prime}} \cdot \mathbf{v}_{n}\right)}{\omega-E_{n} / \hbar}\right] \hat{a}_{\mathbf{k}, s^{\prime}}(0)=0
$$

so that the magnitudes of the wave vectors are the eigenvalues of a $2 \times 2$ matrix, whose elements are functions of the frequency and propagation direction.

It is useful to note that this form, Eq. (7), can also be derived semiclassically, by looking for plane-wave solutions to Maxwell's equations, including the excitonic resonances via a frequency-dependent dielectric function $\epsilon(\omega)$. In an optically isotropic material, $\epsilon(\omega)$ is a scalar, and the polariton dispersion satisfies $c^{2} k^{2} / \omega^{2}=\epsilon(\omega)$ [39]. In the present case, however, the optical response is anisotropic due to the magnetic field, and we must consider the vector equation

$$
-\mathbf{k} \times \mathbf{k} \times \mathbf{E}=\frac{\omega^{2}}{c^{2}} \epsilon(\omega) \mathbf{E} .
$$

Longitudinal modes, with $\mathbf{k} \| \mathbf{E}$, occur if $\epsilon(\omega)=0$. To obtain the equation for the transverse modes, we take matrix elements of Eq. (8) in a basis perpendicular to $\hat{\mathbf{k}}$, such as $\mathbf{e}_{\mathbf{k}, \pm}$. This eliminates the zero eigenvalue of the operator $\mathbf{k} \times \mathbf{k} \times$, i.e., the longitudinal polariton, and gives

$$
\left[\left(\omega^{2}-c^{2} k^{2} / \epsilon\right) \delta_{s s^{\prime}}+\frac{\omega^{2}}{\epsilon} \chi_{s s^{\prime}}\right] \mathbf{E}_{\mathbf{k}, s^{\prime}}=0
$$

where $\chi_{s s^{\prime}}(\omega)=\mathbf{e}_{\mathbf{k}, s}^{\dagger} \chi(\omega) \mathbf{e}_{\mathbf{k}, s^{\prime}}$ is the transverse part of the excitonic susceptibility and $\epsilon$ is the background permittivity. We approximate the prefactors in this expression as $\left(\omega^{2}-\right.$ $\left.c^{2} k^{2} / \epsilon\right) \approx 2 \omega(\omega-c k / \sqrt{\epsilon})$ and $\omega^{2} / \epsilon \approx \omega E_{g} / \hbar \epsilon$. Comparing this expression with Eq. (7), we see that the final term in the latter is related to the susceptibility by

$$
\chi_{s s^{\prime}}=-\frac{\Omega_{0}^{2} \hbar \epsilon}{2 E_{g}} \sum_{n} \frac{\left(\mathbf{e}_{\mathbf{k}, s}^{*} \cdot \mathbf{v}_{n}^{*}\right) \cdot\left(\mathbf{e}_{\mathbf{k}, s^{\prime}} \cdot \mathbf{v}_{n}\right)}{\omega-E_{n} / \hbar} .
$$

The spectrum $k(\omega, \theta, \phi)$ can be found straightforwardly by solving the secular equation for Eq. (7), which is a quadratic in $k$. It may be noted that $\chi_{s s^{\prime}}$, and hence the polariton spectrum, is independent of $\phi$. This reflects the rotational symmetry of the problem about the magnetic field $(\theta=0)$. The combination of the form of Eq. (7) with that of $\chi_{s s^{\prime}}$ imposes an additional symmetry between the solutions at $\theta$ and those at $\pi-\theta$. We may therefore set $\phi=0$ and consider the interval $\theta \in\left[0, \frac{\pi}{2}\right]$. We note that whereas the secular equation for Eq. (7) is a quadratic in $k$, that for $H_{8}$ is an eighth-order polynomial in $\omega$. Thus there are, in general, two wave vectors for each frequency, from the two dispersing photon modes. 


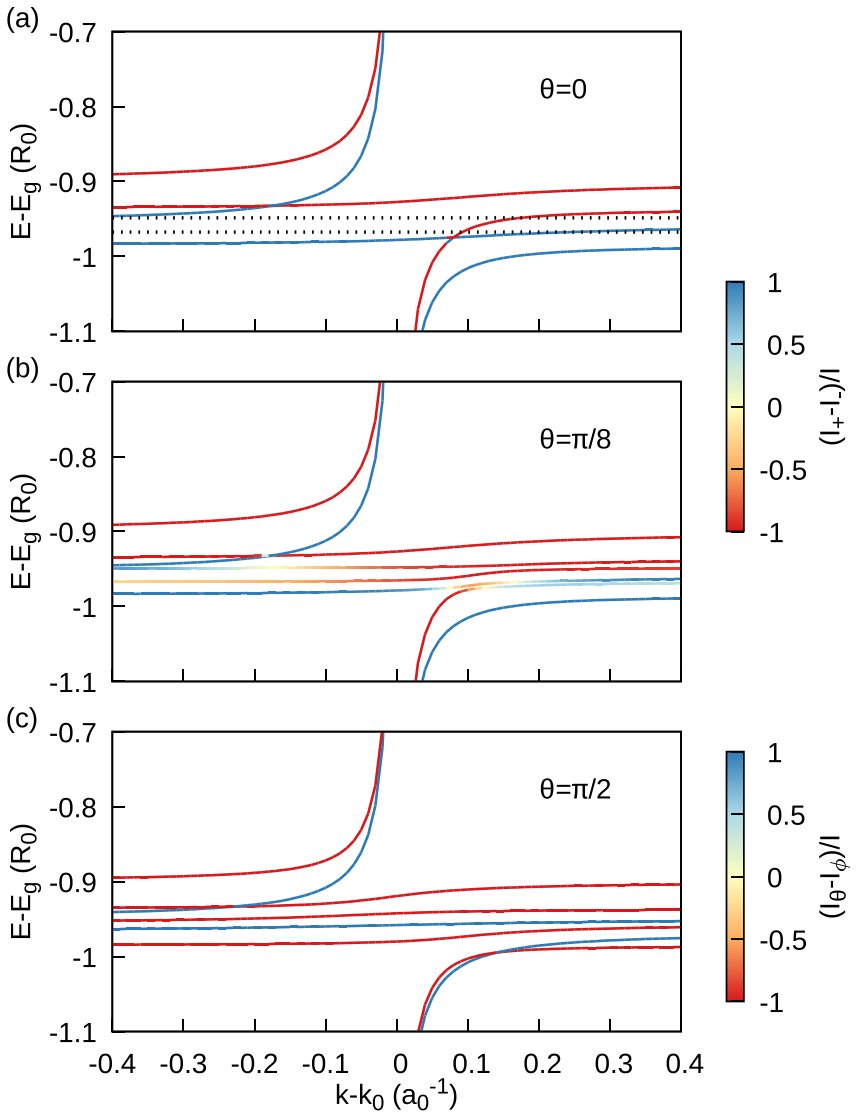

FIG. 2. (a) Solid curves: polariton dispersion relations for GaAs in a magnetic field for $\theta=0$. The coloring shows the degree of circular polarization. Dotted lines: energies of the z-polarized excitons. (b) Polariton dispersion for $\theta=\pi / 8$. (c) Polariton dispersion for $\theta=\pi / 2$. The coloring here shows the degree of linear polarization. Energies are relative to the band gap in units of the exciton Rydberg, and wave vectors are relative to $k_{0}$ in units of the inverse exciton Bohr radius.

There are, however, eight frequencies for each wave vector, from those two photon modes as well as the six nondispersing bright excitons.

\section{RESULTS}

\section{A. Topological singularities: Hermitian case}

Our primary interest is in the degeneracy structure of the magneto-exciton-polariton dispersion relation, which we first consider in the Hermitian case without dissipation. It is possible to make some observations that constrain the possible degeneracies based on the symmetry of the problem. Owing to the $\phi$ invariance of the solutions, we know that propagation in the $\theta=0$ direction is the only configuration for which isolated degeneracies are possible. Correspondingly, if degeneracies occur at any nonzero $\theta$, they are necessarily extended degeneracies over all $\phi$.

In Fig. 2 we plot the dispersion of the transverse modes, obtained from the secular equation for Eq. (7). The three panels refer to propagation along the field, $\theta=0$, at a small angle to it, $\theta=\pi / 8$, and perpendicular to it, $\theta=\pi / 2$. The polarization of the modes is shown by the coloring. Energy is measured relative to the band gap $E_{g}$ and in units of the exciton Rydberg energy. The wave vector is measured relative to $k_{0}=\sqrt{\epsilon}\left(E_{g}-R_{0}\right) / \hbar c$, which is the wave vector at which the bare linear photonic dispersion would cross an unperturbed exciton. The wave vector is measured in units of the inverse exciton Bohr radius.

The spectrum for propagation along the $z$ axis is shown in Fig. 2(a). In this case the two $z$-polarized excitons, $X_{3}$ and $X_{6}$, do not couple to light, and there are only six modes in the transverse spectrum. The other excitons are circularly polarized, and each circular polarization of light mixes with the two excitons of that polarization. This gives rise to a spectrum with a lower, intermediate, and upper branch for each circular polarization. The lower branch begins at low energy as a purely photonic, linearly dispersing, mode, which anticrosses with the lower-energy exciton, asymptoting horizontally to approach that exciton energy at large $k$. Above that energy there is an intermediate branch, which initially has an imaginary $k$ as it lies in the polaritonic (longitudinal-transverse) gap [13]. This mode then becomes a propagating polariton with $k=0$ at the gap edge and then approaches the higher exciton energy at large $k$. Above this there is an upper branch, which again begins as a solution with imaginary $k$, before becoming a propagating solution, and finally approaching the photon dispersion at large $k$.

Figure 2(b) shows the spectrum at a small angle to the $z$ axis. Comparing this spectrum with that in Fig. 2(a), we see that there are now eight branches, because the $z$-polarized excitons now couple to light. Moreover, we find that this spectrum is nondegenerate, with avoided crossings which originate from the degeneracies at $\theta=0$. The two degeneracies in Fig. 2(a) between the different circular modes have split. The splittings are quite small, due to the small angle chosen, but are nonetheless present. In addition, we see that the three intersections between the $z$-polarized excitons and the transverse modes at $\theta=0$ have split, to organize into the two additional transverse branches at $\theta \neq 0$. There is in fact a fourth intersection of this nature, involving the highest-energy $z$-polarized exciton, but it is with an evanescent mode at an imaginary $k$.

Figure 3 shows the dispersion relation near some of these singularities. Since the dispersion has rotational symmetry around $z$, we plot it as a function of $k_{z}$ and the wave vector in the $x y$ plane, $k_{x y}$. One of the degeneracies between the two circular polarization modes seen in Fig. 2(a) is point A in Fig. 3, lying between the orange and blue (second and third from the bottom) sheets. It can be seen to split linearly in $k_{z}$ but quadratically in $k_{x y}$. The same structure appears for the other crossing of the circular modes at $\theta=0$ (not shown). The Hamiltonian close to each of these degeneracies is therefore of the form

$$
c_{1}\left(k_{x}^{2} \sigma_{x}+k_{y}^{2} \sigma_{y}\right)+c_{2}\left(k_{z}-k_{c}\right) \sigma_{z}
$$

for appropriate constants $c_{1}$ and $c_{2}$ and crossing point $k_{c}$. Following the procedure given in Ref. [40], we determine that such a dispersion implies these degeneracies have zero topological charge. However, Fig. 3 also shows three crossings involving the $z$-polarized excitons, labeled B, C, and D. As can be seen in the figure, the dispersion near each of these points forms a pair of touching cones. The energy splitting is 
(a)

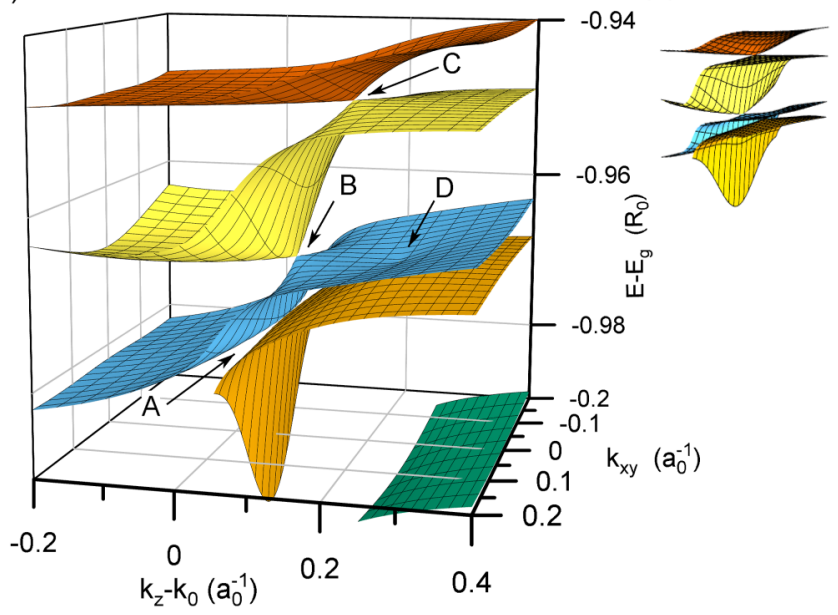

FIG. 3. (a) Polariton dispersion relation for GaAs in a magnetic field, centered around propagation along the magnetic-field direction, $z$. The dispersion relation has rotational symmetry around the $z$ axis. It is shown as a function of $k_{z}$ and the wave vector in the $x y$ plane, $k_{x y}= \pm \sqrt{k_{x}^{2}+k_{y}^{2}}$. Two Weyl points with a linear dispersion in all directions, $\mathrm{B}$ and $\mathrm{C}$, and a band touching with a mixed quadratic/linear dispersion, A, can be seen. A third Weyl point, D, lies between the orange and blue sheets (second and third from the bottom). (b) The same data from a different perspective.

linear in all three components of the wave vector, measured from the degeneracy. This linear splitting is further shown in Fig. 4, and by the analysis below [Eq. (12)]. These points are, therefore, Weyl points. Their linear dispersion implies that they are topologically protected, carry a unit topological charge, and are monopoles of the Berry flux [41,42]. This is because the only form possible for a two-band Hamiltonian, with a degeneracy away from which the bands split linearly in all directions, is $\pm \boldsymbol{\sigma} \cdot\left(\mathbf{k}-\mathbf{k}_{c}\right)$ (up to transformations of the coordinates). This implies topological protection, because any perturbations are additions of the Pauli matrices, which

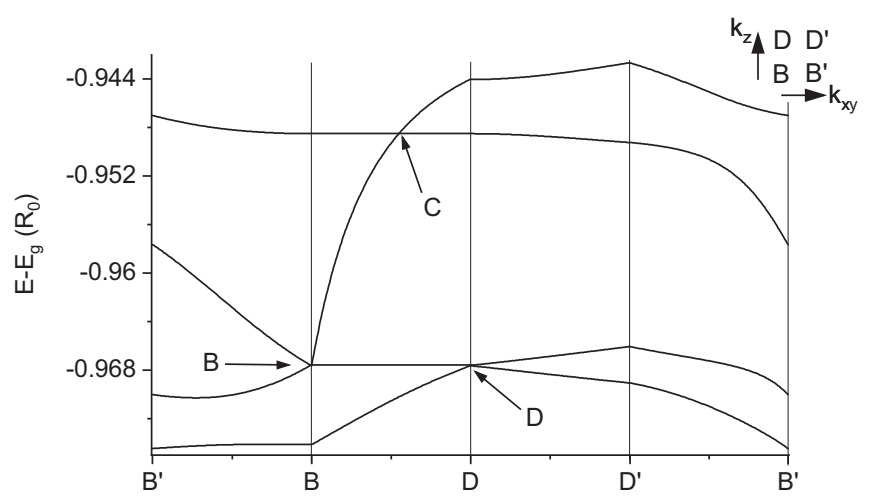

FIG. 4. Polariton dispersion relation for GaAs in a magnetic field, along a square path through $k$ space (horizontal axis). Two corners of the square are the Weyl points marked $B$ and $D$, here and on Fig. 3. The two remaining corners $B^{\prime}$ and $D^{\prime}$ are formed by displacing these points in a direction perpendicular to $k_{z}$, as indicated in the top right of the figure. There is a third Weyl point along one side of the square, marked $\mathrm{C}$. merely move the degeneracy. The unit topological charge can be confirmed using the approach in Ref. [40] as above. The Weyl points B, C, and D correspond to three of the degeneracies between the transverse polaritons and the $z$-polarized excitons at $\theta=0$. The final fourth such degeneracy, lying in one of the longitudinal-transverse gaps [13], is also a Weyl point, with a linear dispersion. However, it occurs at an imaginary $k$.

The dispersion perpendicular to the applied field $(\theta=$ $\pi / 2$ ) is shown in Fig. 2(c). As indicated by the coloring, in this case the transverse modes are purely linearly polarized, along the polar vectors $\mathbf{e}_{\theta}=-\mathbf{z}$ and $\mathbf{e}_{\phi}$. Considering this geometry, we identify two further degeneracies in the polariton dispersion, where modes with these two polarizations cross. These are both extended ring degeneracies, due to the $\phi$ invariance of the system.

We see that there are, in total, eight distinct degeneracies of the polariton dispersion relation in the region $0 \leqslant \theta \leqslant \pi / 2$. Of these eight degeneracies, six are isolated degeneracies occurring in the $\theta=0$ direction, and two are extended degeneracies occurring in the $\theta=\pi / 2$ plane. The six isolated degeneracies divide into four Weyl points, one of which is at an imaginary $k$, and two topologically trivial degeneracies with a mixed quadratic-linear dispersion.

The Weyl points are degeneracies between the z-polarized excitons and the $x y$-polarized polaritons at $\theta=0$. To see why this gives a Weyl point, with a linear dispersion, we note that the coupling between such modes - and hence the splitting of the degeneracy - is proportional to $\sin (\theta)$ [see Eq. (A2)]. This leads to a splitting of the degeneracy which is linear in the magnitude of the transverse wave vector, $\operatorname{since} \sin (\theta) \approx \theta \propto$ $\left|k_{x y}\right|$, where $k_{x y}= \pm \sqrt{k_{x}^{2}+k_{y}^{2}}$. Formally, the Hamiltonian for the two modes near the degeneracy takes the form

$$
\left(\begin{array}{cc}
c^{\prime}\left(k_{z}-k_{c}\right)+\omega_{0} & \Omega \sin (\theta) / 2 \\
\Omega \sin (\theta) / 2 & \omega_{x}
\end{array}\right),
$$

which, with $\sin (\theta) \approx k_{x} / k_{c}$ (for $k_{y}=0$ ), gives a linear dispersion in $k_{x}$ and $k_{z}-k_{c}$ at the degeneracy $\omega_{0}=\omega_{x} . \Omega$ is the strength of the coupling between the $z$-polarized exciton and the polariton, involving the amplitude, in the polariton, of the $\mathbf{e}_{\theta}$-polarized photon. $k_{c}$ is the wave vector at the degeneracy, $\omega_{0}=\omega_{x}=c^{\prime} k_{c}$ is the frequency, and $c^{\prime}$ is the velocity. Notably, these Weyl points lie at the critical tilt between a type-I and a type-II point [43] and as such are the three-dimensional (Weyl) generalization of the recently achieved type-III Dirac point [44]. These classifications distinguish Weyl points based on their isofrequency contours. In type-I cases the energy contour is a point, and in type-II cases the contours are surfaces, while type-III cases represent the transition between type-I and type-II cases where the contour is a line.

\section{B. Exceptional points in the dispersion relation}

In the presence of damping, the Hamiltonian, $H_{8}$, becomes non-Hermitian, and the polariton dispersion, $\omega(k, \theta, \phi)$, can contain rings of exceptional points arising from the Weyl points described above. This can be seen by considering the local Hamiltonian, Eq. (12), for one of the Weyl points. Damping will arise from the phonon or impurity scattering of the 
polariton; exciton losses, through Auger recombination and trapping in dark impurity states; and photon losses, from any background absorption and the escape of light through the boundaries of the sample. Such damping can be modeled by introducing imaginary parts to the frequencies of the corresponding oscillators [13], so that we replace $\omega_{0, x} \rightarrow \omega_{0, x}-$ $i \gamma_{0, x}$ in Eq. (12).

To understand the origins of the exceptional points, we consider the eigenvalues of Eq. (12) at the bare resonance, which is achieved by tuning $k_{z}$ such that the real parts of the diagonal elements are equal. At this point the splitting, in the presence of damping, is $\sqrt{\Omega^{2} \sin ^{2} \theta-\left(\gamma_{0}-\gamma_{x}\right)^{2}}$. Thus the imaginary parts of the eigenvalues are split, and the real parts degenerate, for angles less than $\theta_{c}=\arcsin \left|\gamma_{0}-\gamma_{x}\right| / \Omega$, while the opposite is true for angles greater than this value. This corresponds to the transition between the weak-coupling and strong-coupling regimes for the two modes [12]. In the weak-coupling regime the coupling strength $\Omega \sin \theta$ is smaller than the effective damping rate, and the normal modes are at the original degenerate frequencies, whereas in the strongcoupling regime it is larger, and the normal-mode frequencies (the real parts of the eigenvalues) are split. Because the interaction between the modes depends on the angle, we can use it to access the transition between these two regimes. At the critical angle $\theta_{c}$, marking the onset of strong coupling between these modes, we see that both the real and imaginary parts of the spectrum are degenerate, so we have an exceptional point. Further details of the origins and consequences of such exceptional points, which are generic features of parameterized non-Hermitian eigenvalue problems, can be found in the review by Heiss [6]. In our case the degeneracy occurs at all $\phi$, so that there is a ring of exceptional points in the space of wave vector, where both the real and imaginary parts of the polariton energies are degenerate and, as a corollary [6], the non-Hermitian Hamiltonian is defective.

In Fig. 5 we show an exceptional point of this type, in the spectrum of the full Hamiltonian, $H_{8}$. Figures 5(a) and 5(b) show the real and imaginary parts, respectively, of the energies, and the expected local structure around an exceptional point [6] is clearly visible. As can be seen from the analysis above, the angle of the exceptional point depends on the difference in the damping constants; if this is too small, the Weyl exceptional ring will be indistinguishable from a Weyl point. For Fig. 5 we have chosen to introduce damping $\gamma_{3}=$ $0.01 R_{0} / \hbar$ for the $X_{3}$ exciton only, so that the exceptional point structure is clear. This is a simple, nonrealistic choice, made to illustrate the exceptional points which occur more generally in $H_{8}$ when the modes have differing damping rates. Differences in the damping rates of the different exciton-polariton branches can be expected due to the differing dispersion relations and photon-exciton fractions, and from exciton spin relaxation $[45,46]$ among the Zeeman-split levels.

\section{Exceptional points in the isofrequency surface}

We now consider the effect of damping in terms of the complex-valued wave vector $k(E, \theta, \phi)=k(E, \theta)$ as a function of the real-valued energy and propagation direction. This may be compared with the treatment above, where we considered the complex-valued energy as a function

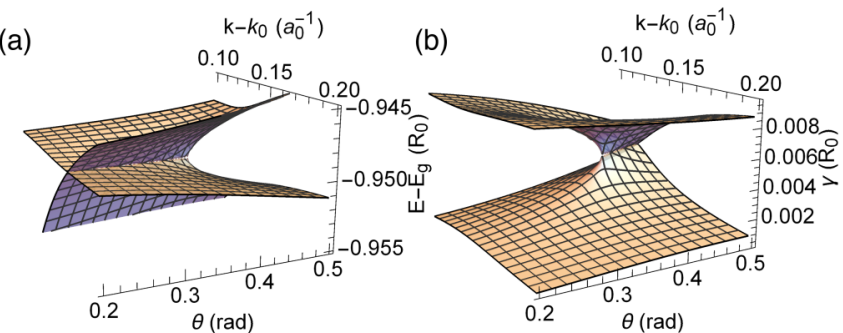

(c)

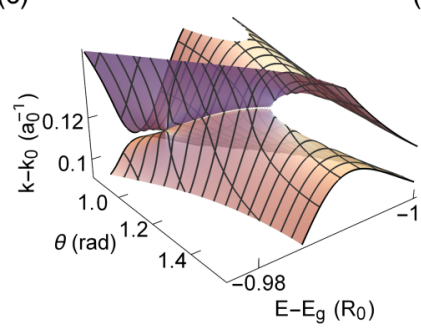

(d)

FIG. 5. Exceptional points in the polariton dispersion (top panels) and wave-vector surface (bottom panels). (a) and (b) Real and imaginary parts of the polariton energies $E(k, \theta)$, for a real wave vector of magnitude $k$, at propagation direction $\theta$. Only one exciton, $X_{3}$, is damped, with rate $\gamma_{3}=0.01 R_{0} / \hbar$. (c) and (d) Real and imaginary parts of the polariton wave vector $k(E, \theta)$, for a real energy $E$. All the excitons have an equal damping rate $\gamma=0.008 R_{0} / \hbar$.

of the real-valued wave vector. At a particular energy the two-sheeted function $k(E, \theta, \phi)$ is an isofrequency surface of the dispersion relation, whose normals give the ray directions [36]. More generally, real energies correspond to monochromatic continuous-wave excitation, and the function $k(E, \theta)$ describes the propagation of polaritons under such conditions. As we shall see, the isofrequency surface can have rings of exceptional points (at particular real energies), similar to those in the dispersion relation (at particular real wave vectors).

Figure 6 shows the complex-valued wave-vector function obtained using the parameters of Fig. 2(a) with damping $\gamma=$ $0.015 R_{0} / \hbar$ for the excitons. Here and throughout this section, we consider an equal damping rate of all the excitons, $\gamma_{n}=\gamma$.

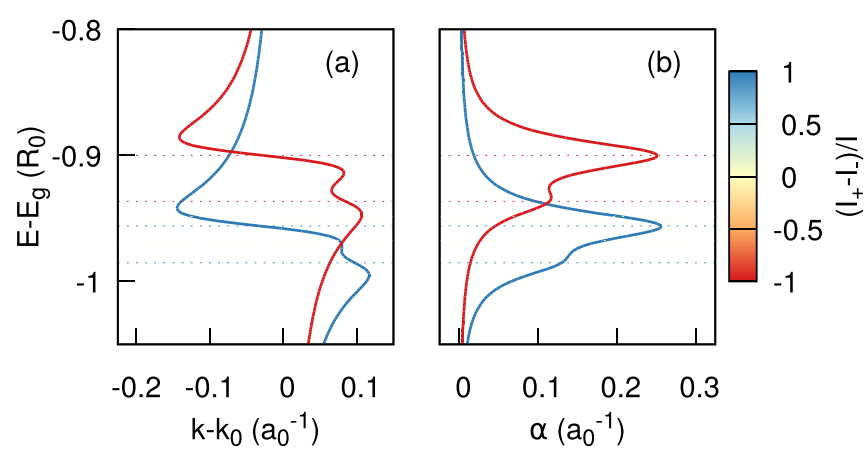

FIG. 6. (a) Real and (b) imaginary parts of the wave vector of the polariton dispersion relation at real energies, for propagation at $\theta=$ 0 . Coloring shows the circular polarization of the modes. Dashed horizontal lines show the energies of the excitons colored by polarization. All excitons have an equal damping rate $\gamma=0.015 R_{0} / \hbar$. 
(a)

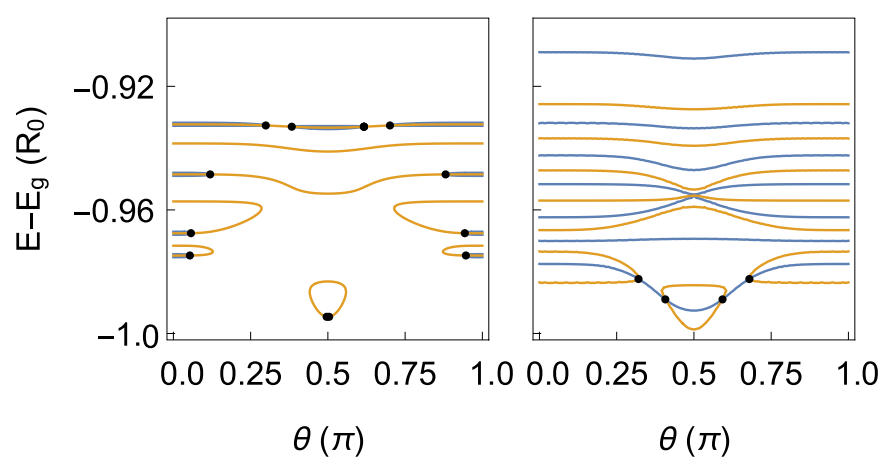

FIG. 7. Exceptional points of the complex wave vector $k(E, \theta)$ for real energies, for two values of the exciton damping: (a) $\gamma=$ $0.0005 R_{0} / \hbar$ and (b) $\gamma=0.008 R_{0} / \hbar$. The blue (orange) curves are the zero contours of the real (imaginary) part of the discriminant for the characteristic equation determining $k$. The points show the locations of the exceptional points.

As can be seen, the damping blurs the distinction between the lower, intermediate, and upper polariton branches, joining them together for each polarization. In the imaginary parts of the wave vectors, i.e., the absorption coefficients, we can see the microstructure of the individual excitonic resonances and their associated oscillator strengths. We see that there are energies where the real parts of the wave vectors for the two polarizations are degenerate, and there are, also, energies where the absorption coefficients are degenerate. These are not exceptional points, however, as the degeneracies in the real and imaginary parts of $k$ occur at different energies.

The exceptional points of the wave-vector function $k(E, \theta)$ are values of $E$ and $\theta$ where, simultaneously, the real parts of $k$ and the imaginary parts of $k$ are degenerate. To identify these degeneracies, we consider the characteristic equation for Eq. (7), which is a quadratic in $k$. The exceptional points are the zeros of the discriminant of this quadratic. They can be found by plotting the zero contours of its real and imaginary parts and looking for their crossings. This is shown for two different values of the damping rate in Fig. 7.

Figure 7(a) shows the situation for a small damping rate, $\gamma=0.0005 R_{0} / \hbar$. This corresponds to an exciton relaxation time $1 / \gamma \approx 300 \mathrm{ps}$, similar to that observed in quantum wells [45]. Figure 7(b) shows the results for stronger damping, $\gamma=0.008 R_{0} / \hbar$, so that $1 / \gamma \approx 20$ ps. In both cases we see that there are exceptional points, which originate from the degeneracies of the polariton dispersion in the absence of damping. As damping is introduced the degeneracies of the polariton dispersion move in the $(\theta, E)$ plane. The richest structure in terms of degeneracies is at low damping, as in Fig. 7(a), where we see there are six exceptional points in the region $0 \leqslant \theta \leqslant \pi / 2$. Since all of these points occur at nonzero $\theta$, they correspond to rings of exceptional points in the isofrequency surface, at certain energies, owing to the $\phi$ independence of the solutions. As the damping is increased the exceptional points annihilate and their number reduces, as can be seen in Fig. 7(b), where there are now only two exceptional points.
Figures 5(c) and 5(d) show the real and imaginary parts of the complex wave vector, as functions of the real energy $E$ and angle $\theta$, in the region containing the two exceptional points of Fig. 7(b). The two exceptional points are joined by a line degeneracy in the real parts of the wave vector, which is clearly visible in Fig. 5(c). The structure around each exceptional point may be compared with an exceptional point in the complex energy [Figs. 5(a) and 5(b)]. We again have the expected general form, i.e., line degeneracies in each of the real and imaginary parts, which meet at the exceptional point. The overall structure may, also, be compared with that described by Berry and Dennis [37] for frequency-independent absorbing dielectrics, for which the complex function $k(\theta, \phi)$ contains degeneracies in particular wave-vector directions. These exceptional points define the "singular axes" of the crystal. They are points in the space of wave-vector direction, but occur at all frequencies. The degeneracies of the complexvalued wave vector described here are, instead, extended in the space of wave-vector direction (forming rings), but occur only at specific frequencies.

\section{CONCLUSION}

The strong coupling of light to excitons in a magnetic field gives rise to topologically nontrivial dispersion relations $\omega(k)$, and wave-vector surfaces $k(E)$, for polaritons in bulk zinc-blende semiconductors. The complex degeneracy structure of the dispersion provides a route to realizing topological effects for polaritons in three dimensions, going beyond previous work in two-dimensional [14-21], and also one-dimensional $[47,48]$, systems such as microcavities. In the absence of dissipation the polariton dispersion contains Weyl points, for propagation along the field, and ring degeneracies, for propagation perpendicular to it. In the presence of dissipation the Weyl points become rings of exceptional points, which generalize the corresponding Dirac exceptional rings of two-dimensional dissipative systems [7]. A realization of Weyl exceptional rings in cold atomic gases has recently been proposed [8]; the present work shows that a different realization in semiconductors may be possible.

Topological bands, Weyl points, and surface states (Fermi arcs) have recently been revealed in transmission experiments $[2,11,24,25,49]$ on photonic band structures [41]. The topological dispersion relations described here, and their consequences, would also give signatures in transmission. Polariton spectra are, however, typically determined using angle-dependent reflectivity measurements. Such techniques are well established [50] and have been used to determine exciton-polariton spectra in bulk semiconductors, with resolution sufficient to resolve Zeeman-split levels [51-55]. The exciton-polariton dispersion gives rise to nonstandard line shapes in reflectivity, so the spectra need to be interpreted by comparison to coupled oscillator models [50]. Another approach would be to pump incoherently above the polariton branches and study the spectra in photoluminescence. This creates the further possibility of exploring the impact of gain on the topological bands and surface modes and creating a polaritonic topological laser based on surface states. Perhaps the most promising way, however, in which the polariton system goes beyond existing photonic topological materials is 
through the presence of large nonlinearities, giving it potential for realizing topological strong-interaction effects using light.

An important question is the extent to which the effects described here can be observed, given realistic values of the dissipation. We see from Figs. 1 and 3 that the scale of the multiple polariton branches is set by the Zeeman splittings in the magnetoexciton spectrum. Note that the light-matter coupling strength, $\hbar \Omega_{0}$, is much larger and therefore not a limiting factor. To observe the multiple polariton bands, along with the Weyl points, will require the exciton damping to be smaller than at least some, or ideally all, of the splittings. The achievability of such a regime is demonstrated by many reflectivity and photoluminescence experiments on zinc-blende semiconductors, in which polaritons formed from
Zeeman-split excitons are resolved [51-55]. In those works some, but not all, of the Zeeman lines are resolved at the relatively low field $B_{\max }$ considered here. However, all of the lines are resolved at higher fields, where the splittings become larger. Although such fields invalidate the perturbative calculation of the exciton energies we took as input to our model, the exciton levels and their polarizations remain. Thus the qualitative features of the polariton spectra predicted by our model will survive, with only quantitative changes.

\section{ACKNOWLEDGMENTS}

We acknowledge support from the Irish Research Council through Award No. GOIPG/2015/3570 and the Science Foundation Ireland through Award No. 15/IACA/3402.

\section{APPENDIX: POLARITON HAMILTONIAN MATRIX}

As discussed in the main text, we obtain the polariton spectrum from the Heisenberg equations of motion for the exciton and photon annihilation operators. In terms of the vector of annihilation operators

$$
\psi=\left(\hat{a}_{+}, \hat{a}_{-}, \hat{x}_{2}, \hat{x}_{3}, \ldots, \hat{x}_{7}\right)^{T}
$$

this reads

$$
i \hbar \frac{d \psi}{d t}=H_{8} \psi=\hbar \omega \psi
$$

where the second equality holds in an eigenstate, in which all the annihilation operators have time dependence $e^{-i \omega t}$. $\hat{a}_{+,-}$ correspond to the two circularly polarized photon modes, and $\hat{x}_{2 \ldots 7}$ correspond to the six optically active exciton modes. The wave-vector labels are suppressed for brevity. The explicit form of the matrix $H_{8}$ is

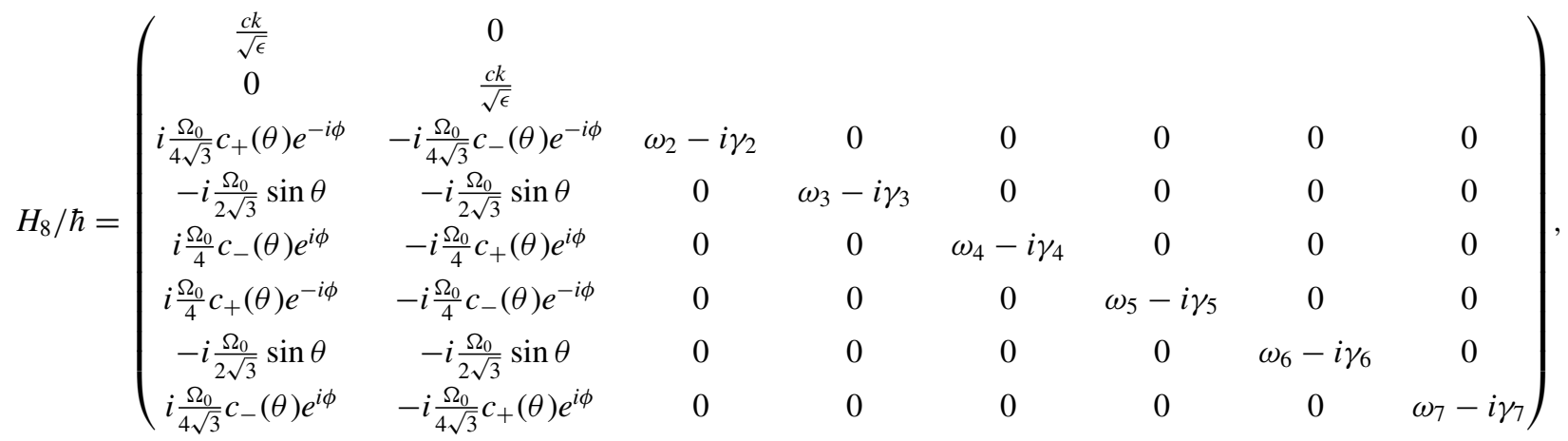

where the omitted parts of the first two rows are Hermitian conjugates of the corresponding parts of the first two columns, $c_{ \pm}(\theta)=(1 \pm \cos \theta)$, and $\omega_{2 \ldots 7}=E_{2 \ldots 7} / \hbar$ are the exciton frequencies. We treat damping by taking the expectation value of Eq. (A1), so that it becomes a set of coupled equations for the complex amplitudes, in which we can introduce imaginary parts $\gamma_{2 \ldots 7}$ in the exciton frequencies as indicated. The now complex polariton frequencies $\omega$ are then computed as the eigenvalues of $H_{8} / \hbar$.

[1] T. Ozawa, H. M. Price, A. Amo, N. Goldman, M. Hafezi, L. Lu, M. C. Rechtsman, D. Schuster, J. Simon, O. Zilberberg, and I. Carusotto, Topological photonics, Rev. Mod. Phys. 91, 015006 (2019).

[2] L. Lu, Z. Wang, D. Ye, L. Ran, L. Fu, J. D. Joannopoulos, and M. Soljačić, Experimental observation of Weyl points, Science 349, 622 (2015).

[3] S.-Y. Xu, I. Belopolski, N. Alidoust, M. Neupane, G. Bian, C. Zhang, R. Sankar, G. Chang, Z. Yuan, C.-C. Lee, S.-M. Huang, H. Zheng, J. Ma, D. S. Sanchez, B. Wang, A. Bansil, F. Chou,
P. P. Shibayev, H. Lin, S. Jia et al., Discovery of a Weyl fermion semimetal and topological Fermi arcs, Science 349, 613 (2015).

[4] H. Shen, B. Zhen, and L. Fu, Topological Band Theory for NonHermitian Hamiltonians, Phys. Rev. Lett. 120, 146402 (2018).

[5] R. El-Ganainy, K. G. Makris, M. Khajavikhan, Z. H. Musslimani, S. Rotter, and D. N. Christodoulides, NonHermitian physics and PT symmetry, Nat. Phys. 14, 11 (2018).

[6] W. D. Heiss, The physics of exceptional points, J. Phys. A: Math. Theor. 45, 444016 (2012). 
[7] B. Zhen, C. W. Hsu, Y. Igarashi, L. Lu, I. Kaminer, A. Pick, S.L. Chua, J. D. Joannopoulos, and M. Soljačić, Spawning rings of exceptional points out of Dirac cones, Nature (London) 525, 354 (2015).

[8] Y. Xu, S.-T. Wang, and L.-M. Duan, Weyl Exceptional Rings in a Three-Dimensional Dissipative Cold Atomic Gas, Phys. Rev. Lett. 118, 045701 (2017).

[9] J. Doppler, A. A. Mailybaev, J. Böhm, U. Kuhl, A. Girschik, F. Libisch, T. J. Milburn, P. Rabl, N. Moiseyev, and S. Rotter, Dynamically encircling an exceptional point for asymmetric mode switching, Nature (London) 537, 76 (2016).

[10] D. Leykam, K. Y. Bliokh, C. Huang, Y. D. Chong, and F. Nori, Edge Modes, Degeneracies, and Topological Numbers in Non-Hermitian Systems, Phys. Rev. Lett. 118, 040401 (2017).

[11] H. Zhou, C. Peng, Y. Yoon, C. W. Hsu, K. A. Nelson, L. Fu, J. D. Joannopoulos, M. Soljačić, and B. Zhen, Observation of bulk Fermi arc and polarization half charge from paired exceptional points, Science 359, 1009 (2018).

[12] A. Kavokin, J. J. Baumberg, G. Malpuech, and F. P. Laussy, Microcavities (Oxford University Press, Oxford, 2017).

[13] P. Yu and M. Cardona, Fundamentals of Semiconductors: Physics and Materials Properties (Springer, New York, 1995).

[14] R. Ge, W. Broer, and T. C. H. Liew, Floquet topological polaritons in semiconductor microcavities, Phys. Rev. B 97, 195305 (2018).

[15] C.-E. Bardyn, T. Karzig, G. Refael, and T. C. H. Liew, Topological polaritons and excitons in garden-variety systems, Phys. Rev. B 91, 161413(R) (2015).

[16] A. V. Nalitov, D. D. Solnyshkov, and G. Malpuech, Polariton $\mathbb{Z}$ Topological Insulator, Phys. Rev. Lett. 114, 116401 (2015).

[17] C. Li, F. Ye, X. Chen, Y. V. Kartashov, A. Ferrando, L. Torner, and D. V. Skryabin, Lieb polariton topological insulators, Phys. Rev. B 97, 081103(R) (2018).

[18] K. Yi and T. Karzig, Topological polaritons from photonic Dirac cones coupled to excitons in a magnetic field, Phys. Rev. B 93, 104303 (2016).

[19] T. Karzig, C.-E. Bardyn, N. H. Lindner, and G. Refael, Topological Polaritons, Phys. Rev. X 5, 031001 (2015).

[20] S. Klembt, T. H. Harder, O. A. Egorov, K. Winkler, R. Ge, M. A. Bandres, M. Emmerling, L. Worschech, T. C. H. Liew, M. Segev, C. Schneider, and S. Höfling, Exciton-polariton topological insulator, Nature (London) 562, 552 (2018).

[21] Y. V. Kartashov and D. V. Skryabin, Two-Dimensional Topological Polariton Laser, Phys. Rev. Lett. 122, 083902 (2019).

[22] T. Gao, E. Estrecho, K. Bliokh, T. Liew, M. Fraser, S. Brodbeck, M. Kamp, C. Schneider, S. Höfling, Y. Yamamoto, F. Nori, Y. S. Kivshar, A. G. Truscott, R. G. Dall, and E. A. Ostrovskaya, Observation of non-Hermitian degeneracies in a chaotic exciton-polariton billiard, Nature (London) 526, 554 (2015).

[23] F. Li, X. Huang, J. Lu, J. Ma, and Z. Liu, Weyl points and Fermi arcs in a chiral phononic crystal, Nat. Phys. 14, 30 (2018).

[24] J. Noh, S. Huang, D. Leykam, Y. D. Chong, K. P. Chen, and M. C. Rechtsman, Experimental observation of optical Weyl points and Fermi arc-like surface states, Nat. Phys. 13, 611 (2017).

[25] B. Yang, Q. Guo, B. Tremain, R. Liu, L. E. Barr, Q. Yan, W. Gao, H. Liu, Y. Xiang, J. Chen, C. Fang, A. Hibbins, L. Lu, and S. Zhang, Ideal Weyl points and helicoid surface states in artificial photonic crystal structures, Science 359, 1013 (2018).

[26] K. Cho, S. Suga, W. Dreybrodt, and F. Willmann, Theory of degenerate $1 s$ excitons in zinc-blende-type crystals in a magnetic field: Exchange interaction and cubic anisotropy, Phys. Rev. B 11, 1512 (1975).

[27] M. Altarelli and N. O. Lipari, Perturbation-theory investigation of the exciton ground state of cubic semiconductors in a magnetic field, Phys. Rev. B 7, 3798 (1973).

[28] R. Winkler, Spin-Orbit Coupling Effects in Two-Dimensional Electron and Hole Systems (Springer, New York, 2003).

[29] H. Fu, L.-W. Wang, and A. Zunger, Excitonic exchange splitting in bulk semiconductors, Phys. Rev. B 59, 5568 (1999).

[30] C. Schäfer, M. Ruggenthaler, V. Rokaj, and A. Rubio, Relevance of the quadratic diamagnetic and self-polarization terms in cavity quantum electrodynamics, ACS Photonics 7, 975 (2020).

[31] Electron-hole exchange mixes different electron-hole spin configurations within each polarization, i.e., total spin $m_{s}+m_{h}$, modifying the oscillator strength and hence producing small changes in the spectrum. At the field $B_{\max }$ the dominant component corresponds to between 93 and $99 \%$ of the wave function, depending on the exciton.

[32] K. Cho, Unified theory of symmetry-breaking effects on excitons in cubic and wurtzite structures, Phys. Rev. B 14, 4463 (1976).

[33] P. Basu, Theory of Optical Processes in Semiconductors (Clarendon, Oxford, 1997).

[34] R. Willardson and A. Beer, Optical Properties of III-V Compounds, Semiconductors and Semimetals Vol. 3 (Academic, New York, 1967).

[35] L. Świerkowski, $1 s$ and $2 s$ states of direct exciton in a magnetic field, Nuovo Cimento B 29, 340 (1975).

[36] M. Born and E. Wolf, Principles of Optics (Cambridge University Press, Cambridge, 1959).

[37] M. V. Berry and M. R. Dennis, The optical singularities of birefringent dichroic chiral crystals, Proc. R. Soc. London, Ser. A 459, 1261 (2003).

[38] K. E. Ballantine, J. F. Donegan, and P. R. Eastham, Conical diffraction and the dispersion surface of hyperbolic metamaterials, Phys. Rev. A 90, 013803 (2014).

[39] H. Haug and S. Koch, Quantum Theory of the Optical and Electronic Properties of Semiconductors (World Scientific, River Edge, 2004).

[40] M.-C. Chang and M.-F. Yang, Chiral magnetic effect in the absence of Weyl node, Phys. Rev. B 92, 205201 (2015).

[41] L. Lu, L. Fu, J. D. Joannopoulos, and M. Soljačić, Weyl points and line nodes in gyroid photonic crystals, Nat. Photonics 7 , 294 (2013).

[42] N. P. Armitage, E. J. Mele, and A. Vishwanath, Weyl and Dirac semimetals in three-dimensional solids, Rev. Mod. Phys. 90, 015001 (2018)

[43] Z. Jalali-Mola and S. A. Jafari, Electrodynamics of tilted Dirac and Weyl materials: A unique platform for unusual surface plasmon polaritons, Phys. Rev. B 100, 205413 (2019).

[44] M. Milićević, G. Montambaux, T. Ozawa, O. Jamadi, B. Real, I. Sagnes, A. Lemaître, L. Le Gratiet, A. Harouri, J. Bloch, and A. Amo, Type-III and Tilted Dirac Cones Emerging from Flat Bands in Photonic Orbital Graphene, Phys. Rev. X 9, 031010 (2019). 
[45] M. Z. Maialle, E. A. de Andrada e Silva, and L. J. Sham, Exciton spin dynamics in quantum wells, Phys. Rev. B 47, 15776 (1993).

[46] I. A. Shelykh, A. V. Kavokin, and G. Malpuech, Spin dynamics of exciton polaritons in microcavities, Phys. Status Solidi B 242, 2271 (2005).

[47] D. D. Solnyshkov, A. V. Nalitov, and G. Malpuech, KibbleZurek Mechanism in Topologically Nontrivial Zigzag Chains of Polariton Micropillars, Phys. Rev. Lett. 116, 046402 (2016).

[48] C. A. Downing, T. J. Sturges, G. Weick, M. Stobińska, and L. Martín-Moreno, Topological Phases of Polaritons in a Cavity Waveguide, Phys. Rev. Lett. 123, 217401 (2019).

[49] W.-J. Chen, M. Xiao, and C. T. Chan, Photonic crystals possessing multiple Weyl points and the experimental observation of robust surface states, Nat. Commun. 7, 13038 (2016).
[50] D. D. Sell, S. E. Stokowski, R. Dingle, and J. V. DiLorenzo, Polariton reflectance and photoluminescence in high-purity GaAs, Phys. Rev. B 7, 4568 (1973).

[51] F. Willmann, S. Suga, W. Dreybrodt, and K. Cho, Magnetoreflectance of the $1 \mathrm{~S}$ exciton ground states in InP and GaAs, Solid State Commun. 14, 783 (1974).

[52] S. B. Nam, D. C. Reynolds, C. W. Litton, R. J. Almassy, T. C. Collins, and C. M. Wolfe, Free-exciton energy spectrum in GaAs, Phys. Rev. B 13, 761 (1976).

[53] S. B. Nam, D. C. Reynolds, C. W. Litton, T. C. Collins, P. J. Dean, and R. C. Clarke, Free-exciton energy spectrum in InP in a magnetic field, Phys. Rev. B 13, 1643 (1976).

[54] Y. Chen, B. Gil, H. Mathieu, and J. P. Lascaray, Excitonpolaritons in InP: Magnetoreflectance investigation, Phys. Rev. B 36, 1510 (1987).

[55] R. Dingle, Magneto-optical investigation of the free-exciton reflectance from high-purity epitaxial GaAs, Phys. Rev. B 8, 4627 (1973). 